medRxiv preprint doi: https://doi.org/10.1101/19005611; this version posted September 11, 2019. The copyright holder for this preprint (which

was not certified by peer review) is the author/funder, who has granted medRxiv a license to display the preprint in perpetuity.

All rights reserved. No reuse allowed without permission.

\title{
A deep learning approach with event-related spectral EEG data in attentional deficit
}

\section{hyperactivity disorder}

3

4 Laura Dubreuil-Vall ${ }^{1,2,3,}{ }^{*}$, Giulio Ruffini ${ }^{3}$, Joan A. Camprodon ${ }^{1}$

5

$6 \quad{ }^{1}$ Department of Psychiatry, Massachusetts General Hospital, Harvard Medical School

7 Laboratory for Neuropsychiatry and Neuromodulation

$8 \quad 14913^{\text {th }}$ st. $2^{\text {nd }}$ floor.

9 Boston, MA 02129

${ }^{2}$ Department of Psychiatry and Clinical Psychobiology, Universitat de Barcelona

08036 Barcelona

14 Spain

15

$16{ }^{3}$ Neuroelectrics Corporation

17210 Broadway, Suite 201

18 Cambridge, MA, 02139

19 USA

20

$21 *$ Corresponding author

22 Laura Dubreuil-Vall, M.Sc.

23 Laboratory for Neuropsychiatry and Neuromodulation

24 Department of Psychiatry

25 Massachusetts General Hospital

26 Harvard Medical School

$27 \quad 14913^{\text {th }}$ st. $2^{\text {nd }}$ floor.

28 Boston, MA 02129

29 Email: 1dubreuilvall@mgh.harvard.edu

30 Phone: +16173906447 
32 Attention deficit hyperactivity disorder (ADHD) is a heterogeneous neurodevelopmental disorder that affects $5 \%$ 33 of the pediatric and adult population worldwide. The diagnosis remains essentially clinical, based on history and 34 exam, with no available biomarkers. In this paper, we describe a deep convolutional neural network (DCNN) for 35 ADHD classification derived from the time-frequency decomposition of electroencephalography data (EEG), 36 particularly of event-related potentials (ERP) during the Flanker Task collected from 20 ADHD adult patients and 3720 healthy controls (HC). The model reaches a classification accuracy of $88 \%$, superior to resting state EEG 38 spectrograms and with the key advantage, compared with other machine learning approaches, of avoiding the need 39 for manual selection of EEG spectral or channel features. Finally, through the use of feature visualization 40 techniques, we show that the main features exciting the DCNN nodes are a decreased power in the alpha band and

41 an increased power in the delta-theta band around 100ms for ADHD patients compared to HC, suggestive of 42 attentional and inhibition deficits, which have been previously suggested as pathophyisiological signatures of 43 ADHD. While confirmation with larger clinical samples is necessary, these results highlight the potential of this 44 methodology to develop CNS biomarkers of practical clinical utility. 


\section{Introduction}

49 deficits in attention, impulsivity (motor and non-motor) and executive dysfunction. It is associated with high

50 morbidity and disability ${ }^{1,2}$, and affects up to $5 \%$ of adults worldwide ${ }^{3-5}$. The diagnosis of ADHD remains

51 essentially clinical, based on history and exam. It can be supported by neuropsychological assessments, but given

52 the heterogeneous cognitive profiles in patients with ADHD, these provide a supportive, not fully diagnostic,

53 function. Significantly, there are many different conditions that present with disordered attention, impulsivity and

54 dysexecutive syndromes, and the range of normal cognitive profiles with variable strengths and weaknesses in

55 these domains is wide, often complicating the differential diagnosis. Hence, a biomarker to reduce the inherent

56 uncertainty of clinical diagnosis would be of great value.

58 Electroencephalographic (EEG) signals contain rich information associated with functional dynamics in the brain.

59 The use of EEG in ADHD began more than 75 years ago with Jasper et al. ${ }^{6}$ reporting an increase in the EEG

60 power of low frequencies in fronto-central areas. Since then, human electrophysiological studies using EEG

61 spectral analyses and event-related potentials (ERPs) have established relevant signatures of executive dysfunction

62 in $\mathrm{ADHD}^{7}$. In contrast to spontaneous EEG, ERPs reflect changes in the electrical activity of the brain that are

63 time-locked to the occurrence of a specific event, that is, a response to a discrete external stimulus or an internal

64 mental process ${ }^{8}$. ERPs also provide non-invasive neurophysiological measurements with high temporal resolution,

65 allowing to assess dysfunctional brain dynamics, including cognitive processes that may not be apparent at the

66 behavioral level ${ }^{9,10}$. Indeed, ERPs are commonly used clinically in neurophysiological diagnostic units to support

67 the assessment of neuropsychiatric disorders (e.g., multiple sclerosis ${ }^{11}$ ) and sensory disorders (e.g., screening of 68 neonates for hearing impairments ${ }^{12}$ ).

70 Artificial neural networks (ANNs) have recently become a promising application of artificial intelligence (AI) in

71 healthcare ${ }^{13}$. Machine learning, a subtype of AI, and deep learning, a specialized sub-field of machine learning,

72 have been increasingly used in clinical research with promising results. Machine learning can be described as the

73 practice of using algorithms to train a system by using large amounts of data, with the goal of giving it the ability

74 to learn how to perform a specific task, and then make an accurate classification or prediction. Deep learning is a

75 subset of machine learning algorithms that break down the tasks in smaller units (neural networks, NNs) often 
76 providing higher levels of accuracy.

78 NNs are characterized by their network architecture, defined by the anatomical layout of its connected processing 79 units, the artificial "neurons", according to a loss or optimization function that specifies the overall goal of the 80 learning process. Connections are "trained", or taught how to do the desired task, by using a training algorithm 81 that iteratively changes parameters of the NN such that the target function is ultimately optimized based on the 82 inputs the NN receives. There are different types of NNs with different designs and architectures derived from 83 different principles, or conceived for different purposes. The most basic ones are the feed-forward NNs (FNNs), in

84 which activity is propagated unidirectionally layer-by-layer from the input up to the output stage, with no feedback 85 connections within or between layers. We have previously used a specific type of FFNs (feed-forward 86 autoencoders) for the analysis of EEG data with promising outcomes ${ }^{14}$. Recurrent Neural Networks (RNN) are 87 another type of NN that, unlike FFNs, are based on architectures with feedback ("recurrent") connections within or 88 between layers. In related work, we used Echo State Networks (ESNs), a particular type of RNN, to classify 89 Parkinson patients from HC using EEG time-frequency decompositions ${ }^{15}$ with successful results. The main 90 limitation of RNNs is, however, their computational $\operatorname{cost}^{16}$. In addition, one of the main critics to deep NN is their 91 "black-box" nature, i.e., the difficulty in tracing a prediction back to which features are important and 92 understanding how the network reached the final output, which will be later addressed in this study.

94 Previous studies have successfully classified ADHD patients from HC using machine learning techniques with 95 accuracies of more than $90 \%{ }^{17-23}$, but the selection of disease-characterizing features from EEG was done 96 manually after an extensive search in the frequency or time domain. However, EEG signals exhibit non-linear 97 dynamics (chaotic signals that do not behave linearly and cannot be represented as combination of basic sub98 signals) and non-stationarity across temporal scales (signals with a mean and variance that do not stay constant 99 over time) that cannot be studied properly using classical machine learning approaches. There is a need for tools 100 capable of capturing the rich spatiotemporal hierarchical structures hidden in these signals. In a previous study ${ }^{24}$,

101 we trained a machine learning system with pre-defined complexity metrics of time-frequency decompositions of 102 EEG data that showed statistically significant differences between REM Sleep Behavior Disorder (RBD) patients 103 and HC, indicating that such metrics may be useful for classification or scoring. While this approach is useful in 104 several domains, it would be advantageous to use methods where the relevant features are found directly by the 105 algorithms instead of pre-defining them manually. 
107 With the goal of building a discrimination system that can classify ADHD patients from HCs, here we explore a 108 deep learning approach inspired by recent successes in image classification using Deep Convolutional Neural 109 Networks (DCNNs), a particular type of NN designed to exploit compositional and translationally invariant 110 features in the data that are present in EEG, i.e., features that are recognizable even if their appearance varies in 111 some way ${ }^{16}$. These networks were originally developed to deal with image data (2D arrays) from different 112 channels or audio data ${ }^{25}$, and more recently, EEG data ${ }^{26,27}$. Similarly, here we train a DCNN with multi-channel 113 two-dimensional time-frequency maps (spectrograms or 2D time-frequency maps), representing EEG spectral 114 dynamics as images with the equivalent image depth provided by multiple EEG channels. These networks treat 115 EEG-channel data as an audio file, and our approach mimics similar uses of deep networks in that domain. 116 Specifically, we use a similar strategy as the one presented by Ruffini et al. ${ }^{28}$, but instead of using spontaneous 117 EEG spectrograms, we use ERP spectrograms (also called Event-Related Spectral perturbation, ERSP) recorded 118 during a Flanker-Eriksen Task (EFT), a well-established experimental task to assess sustained attention, conflict 119 monitoring and response inhibition. Our assumption is that relevant qualities of ERP data are contained in 120 compositional features embedded in this time-frequency representation. Particularly, we expect that DCNNs may 121 be able to efficiently learn to identify features in the time-frequency domain associated to event-related bursting 122 across frequency bands that may help separate classes, similar to what is known as "bump analysis"29. For 123 comparison purposes, we also trained a RNN based on Long Short-Term Memory (LSTM) networks, which can

124 learn long sequences of data but require higher computational demands, and a Shallow Neural Network (SNN) as 125 a baseline, a more basic type of network with only one layer. We also compared the performance of the ERSP data 126 with a dataset of spontaneous EEG data recorded while the participants were at resting state. Lastly, we propose 127 the utilization of deep learning visualization techniques for the mechanistic interpretation of results, particularly

128 the method popularly known as DeepDream ${ }^{30}$. This is important to identify pathophysiological features driving the 129 translational and clinical value of the application, and for the optimized further development and acceptance of 130 such techniques in the clinical domain, where black-box approaches have been extensively criticized.

\section{$132 \underline{\text { Methods }}$}

133 Participants

134 A total of 40 participants including 20 healthy adults (10 males, 10 females) and 20 ADHD adult patients (10 135 males, 10 females) participated in the present study (Table 1). The inclusion criteria for ADHD patients consisted 
136 of a diagnosis of ADHD made by a board-certified clinician according to the Diagnostic and Statistical Manual of

137 Mental Disorders, Fifth Edition (DSM-5) ${ }^{31}$. Symptom profiles and severity were assessed with the Adult ADHD

138 Self-Report Scale (ASRS-v1.1) ${ }^{32}$. Patients were either off stimulant medications or, if undergoing treatment with

139 stimulants, were asked to discontinue two days prior to the experiment, under a physician-guided protocol, and

140 allowed to resume afterwards. Psychiatric comorbidities were allowed as long as ADHD was the primary

141 diagnosis. Psychosis, bipolar disorder, substance use disorder and neurological conditions were exclusion criteria.

142 Healthy participants were included if they did not have any psychiatric or neurologic condition and were not

143 taking any psychoactive medications. All participants gave informed and written consent for participation. The

144 study was approved by the Partners HealthCare System's Institutional Review Board and all experiments were

145 performed in accordance with relevant guidelines and regulations at Massachusetts General Hospital.

Table 1. Participant characteristics

\begin{tabular}{|c|c|c|c|}
\hline & $\operatorname{ADHD}(n=20)$ & HC $(n=20)$ & Significance \\
\hline Demographic & mean $(\mathrm{SD}) *$ & mean $(\mathrm{SD}) *$ & p value ( $T$ test) \\
\hline Age & $43.85(14.78)$ & $29.90(10.77)$ & 0.0006 \\
\hline Females & $10(50 \%)$ & $10(50 \%)$ & 0.5 \\
\hline \multicolumn{4}{|l|}{ Baseline Scores } \\
\hline ASRS & $62.6(9.17)$ & $36.47(11.33)$ & $<0.0001$ \\
\hline \multicolumn{4}{|c|}{ Current medications - N (\%) } \\
\hline No medication & $11(55 \%)$ & & \\
\hline Adderall & $2(15 \%)$ & & \\
\hline Vyvanse & $2(10 \%)$ & & \\
\hline Concerta & $1(5 \%)$ & & \\
\hline Verapamil & $1(5 \%)$ & & \\
\hline Aspirin & $1(5 \%)$ & & \\
\hline Levothyroxine & $1(5 \%)$ & & \\
\hline Modafinil & $1(5 \%)$ & & \\
\hline
\end{tabular}

Abbreviations. SD: Standard Deviation

147 (*) All figures are mean (Standard Deviation) unless otherwise specified.

\section{Experimental Task: Eriksen-Flanker task (EFT)}

150 Each patient underwent three identical experimental sessions separated by 1-2 weeks in which they performed the

151 Eriksen-Flanker task (EFT) (Figure 1) while EEG data was recorded. The EFT is a classic behavioral paradigm in

152 which subjects must attend and respond to the direction of a central arrow that is surrounded ("flanked") by 153 distracting stimuli. The flanking arrows can either have the same (congruent trials) or opposing (incongruent trials)

154 orientation as the central one. Participants are instructed to press the left or right arrow buttons in a keyboard 155 following the direction of the central arrow, ignoring the flankers. In this study there were a total of 140 trials, and 156 each subject had a different, fully random sequence of congruent and incongruent trials, with 2 congruent trials for 
157 each incongruent trial, in order to build a tendency towards the prepotent congruent responses and thus increase

158 the difficulty of conflict detection in incongruent trials. Only incongruent trials were used for classification

159 purposes, as they are the ones that most elicit the conflict-related ERP components that characterize the executive

160 function subtasks of selective attention, inhibition and cognitive control ${ }^{33}$, primarily impaired in ADHD. The

161 accuracy (percentage of correct/incorrect responses) and the reaction time (RT) were measured for each trial,

162 while also recording EEG data during the task. RT of single trials was introduced into a Generalized Linear Model

163 with Mixed Effects (GLMM) with a Gamma distribution, with Group as a fixed factor (ADHD/HC) and Subject

164 ID as a random intercept. Accuracy was also modeled using a generalized logistic regression model with mixed 165 effects and a binomial distribution.

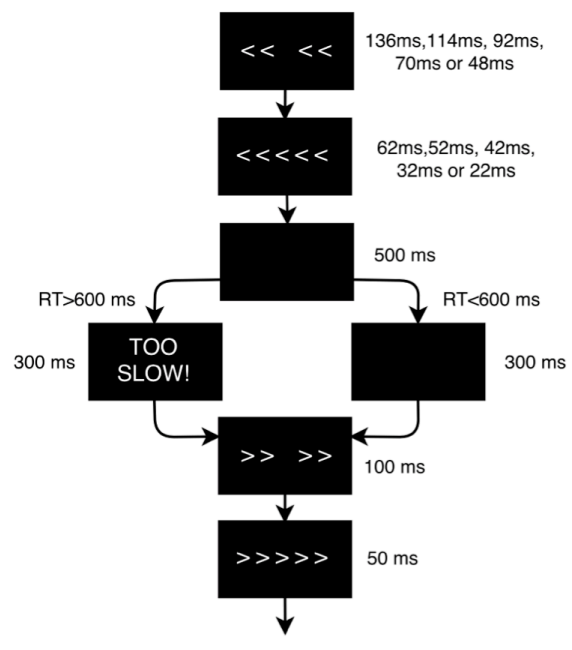

168 Figure 1. Flanker task design scheme. The flanker arrows were first presented alone for duration of $136 \mathrm{~ms}, 114 \mathrm{~ms}, 92 \mathrm{~ms}$,

$16970 \mathrm{~ms}$ or $48 \mathrm{~ms}$ depending on the baseline performance of each subject, and were then joined by the target arrow for $62 \mathrm{~ms}$,

$17052 \mathrm{~ms}, 42 \mathrm{~ms}, 32 \mathrm{~ms}$ or $22 \mathrm{~ms}$, respectively (the duration of the stimuli was adjusted to the psychometric spot in which each

171 subject reached a performance of $80-85 \%$ ). Stimulus presentation was followed by a black screen for $500 \mathrm{~ms}$. The time-

172 window for participants' response was $600 \mathrm{~ms}$ after target onset. If the participant did not respond within the response window,

173 a screen reading 'TOO SLOW!' was presented for $300 \mathrm{~ms}$. Participants were told that if they saw this screen, they should speed

174 up. If a response was made before the deadline, the 'TOO SLOW!' screen was omitted and the black screen remained on screen

175 for the $300 \mathrm{~ms}$ interval. Finally, each trial ended with presentation of the fixation cross for an additional randomly chosen

176 duration $(200,300$ or $400 \mathrm{~ms})$ in order to avoid any habituation or expectation by the subject. Thus, trial duration varied 177 between $1070-1400 \mathrm{~ms}$. 
180 EEG was recorded with the Starstim system (Neuroelectrics, Cambridge, MA, USA) from 7 positions covering the

181 primary hubs of the fronto-parietal executive control network (Fp1, Fp2, F3, Fz, F4, P3 and P4) with $3.14 \mathrm{~cm}^{2}$

$182 \mathrm{Ag} / \mathrm{AgCl}$ electrodes and digitalized with 24-bit resolution at a sampling frequency of $500 \mathrm{samples} / \mathrm{second}$. EEG

183 data was referenced to the right mastoid. Independent component analysis (ICA) was utilized to identify and

184 remove activity associated with blinks, eye movements, and other artifacts. Data was filtered from $1 \mathrm{~Hz}$ to $20 \mathrm{~Hz}$ to

185 remove non-neural physiological activity (skin/sweat potentials) and noise from electrical outlets. Trials were

186 epoched within a time frame of $200 \mathrm{~ms}$ before and $800 \mathrm{~ms}$ after the stimulus onset. The mean of the pre-stimulus

187 baseline $[-200,0] \mathrm{ms}$ was then subtracted from the entire ERP waveform for each epoch to eliminate any voltage

188 offset.

190 To create the ERP spectrograms (or ERSP), the Wavelet transform was applied to each singe trial as implemented

191 in EEGlab's newtimef function, with 1 wavelet cycle at the lowest frequency to 10 cycles at the highest, leading to

19222 frequency bins logarithmically spaced in the $[3,20] \mathrm{Hz}$ range and 20 linear time bins in the $[0,800] \mathrm{ms}$ range,

193 where 0 represents the onset of the target stimuli in incongruent trials. The input data frames were thus

194 multidimensional arrays of the form [22 Frequency bins] x [20 Time bins] x [7 channels], with 3 minutes of data

195 per subject approximately. For comparison purposes, we also processed with the same parameters a dataset of

196 spontaneous EEG data recorded while the same subjects and ADHD patients were resting with eyes closed (no

197 cognitive task performed).

199 Neural network architecture

200 The DCNN, implemented in Tensorflow ${ }^{34}$, is a relatively simple four layer convolutional network, as shown in

201 Figure $2 \mathrm{a}$. In order to avoid overfitting the data (i.e., overtraining the system to the extent that it negatively

202 impacts the performance of the model on new data), we used the so-called "Dropout" method, a regularization

203 technique in which that randomly selected neurons are ignored during training ${ }^{35}$. The number of iterations in the

204 training process was also limited to the point after which more iterations did not improve training significantly and

205 may lead to overfitting, a method known as "early stopping"36. The patch size of the convolutional filter, the

206 pooling parameters and the number of hidden units indicated in Figure 2, as well as the Stochastic Gradient

207 Descent hyper-parameters (number of steps $=600$, batch size $=32$ ), were determined from our previous work using

208 EEG spectrograms ${ }^{28}$, but no fine-tuning or optimization of parameters was applied. 
210 We compared the DCNN's performance with a Shallow Neural Network (SNN) (Figure 2c), a more basic machine 211 neural network with a hidden layer, and with a RNN consisting of stacked LSTM ${ }^{16,37}$, a type of RNN capable of 212 using information about events in the past (memory) to inform predictions in the future (Figure 2b).

213

214 a) DCNN

215

216

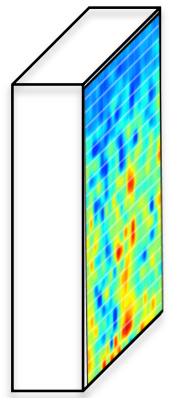

Input ERSP stack

$22 \times 20 \times 7$

Convolution filter: patch size for time and frequency $=3$, strides for time and frequency=3, depth=16.

Pooling: pool size for time and

frequency $=1$, strides for time and frequency=1. Dropout probability $=0.7$

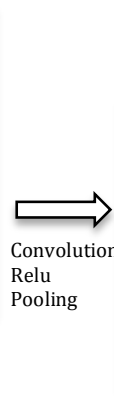

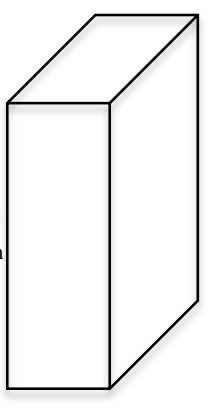

Feature map $22 \times 20 \times 16$ Convolution filter patch_size_frequency $=22$, atch_size_time $=4$, strides_frequency $=22$, strides_time $=1$, depth $=32$. Pooling: pool_strides_frequency $=1$, pool_strides_time $=4$. Dropout probability $=0.7$

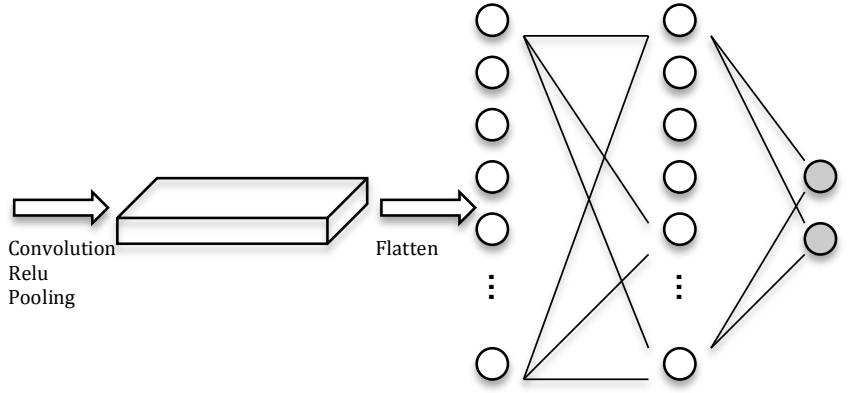

Feature map 128 hidden units, 64 hidden units, Output $1 \times 5 \times 32$ same padding, same padding, (softmax) Dropout pr Dropout prob $=0.8$

c) $\mathrm{SNN}$
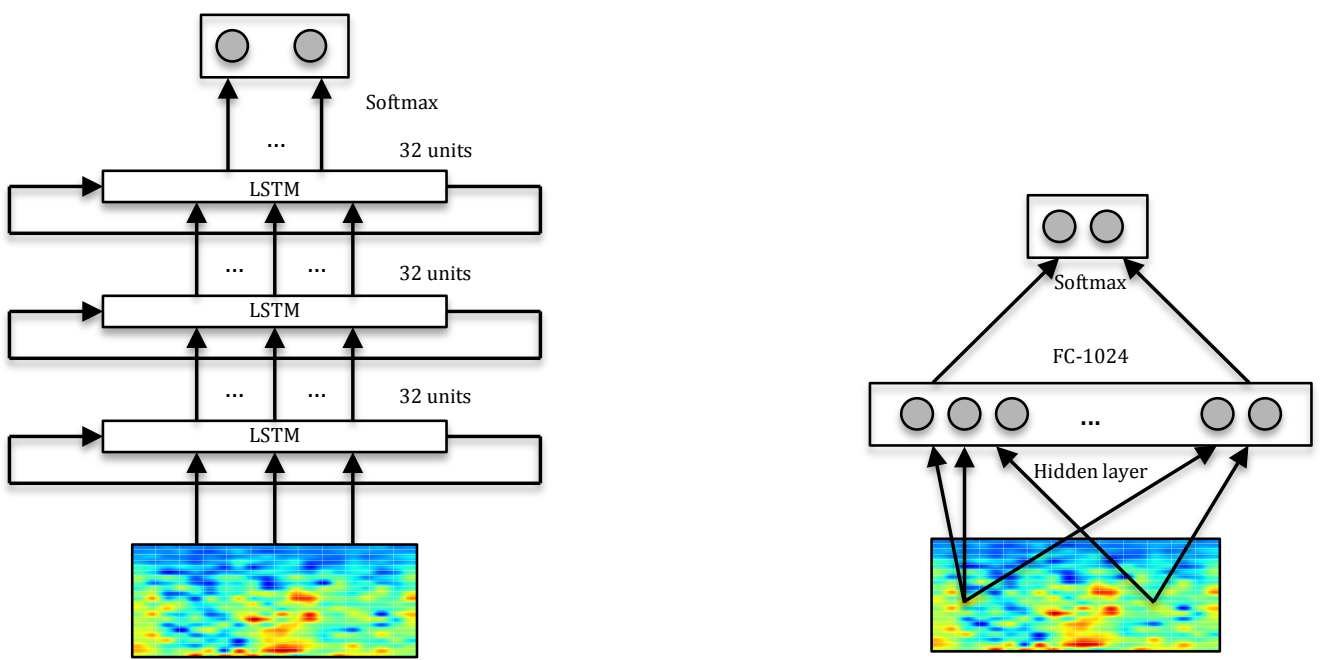

221 Figure 2. Network architectures. a) DCNN model displaying input, convolution with pooling layers, and hidden-unit layers.

222 The first two layers perform the convolution, the Rectified Linear Units (ReLU) function and the pooling processes for feature extraction. The last two layers with 128 and 64 hidden nodes perform the class classification in HC or ADHD. For each trial (or

224 frame), the classifier outputs the probability of the frame belonging to each class (using the softmax function ${ }^{16}$ ) and, after 225 averaging over frames per subject, we obtained the probability of the subject belonging to each class. Classification was performed by choosing the class with maximal probability. b) RNN consisting of three stacked layers of LSTM cells, where 
228 SNN architecture used for comparison with one layer of 1024 units.

$\underline{\text { Performance assessment }}$

231 The performance metrics assessed for each architecture were accuracy (probability of good a classification) and

232 area under the curve (AUC) ${ }^{38}$. Classification performance was validated using the leave-pair out cross-validation

233 (LPO), a method for model selection and performance assessment of deep learning algorithms that consists of

234 training the network $\mathrm{N}=20 \times 20=400$ times (all possible combinations of pairing $1 \mathrm{HC}$ with $1 \mathrm{ADHD}$ ), holding one

235 sample from each group out from the training set at a time, and measuring the performance using the held out pair

236 as a test $\operatorname{set}^{38}$.

238 To account for the significant differences in age between the ADHD and HC groups, we applied the Inverse

239 Probability Weighting (IPW) method $^{39}$, which assigns different weights to the subjects in the training process

240 according to their propensity score ${ }^{40}$. The IPW method lead to the same performance without adjustment in all

241 architectures, thus ruling out the effect of age as a confounding factor.

243 Feature visualization

244 Once the network was trained, it was used to find out what type of inputs optimally excite the output nodes using a 245 method popularly known as "DeepDream", which refers to the generation of synthetic images that produce desired 246 activations in a trained deep network by exaggerating small features within them ${ }^{30}$. The algorithm maximizes a 247 particular class score using gradient descent, starting from a null or random noise image. In particular, we 248 computed the DeepDream spectrograms averaged over $\mathrm{N}=400$ experiments by maximizing the output logits after

24930 iterations in steps of 1, initializing with different random images (seeds).

252 The results from classification using different methods and datasets are detailed in Figure 3a and 3b, showing that 253 the DCNN trained with ERSPs reached an accuracy of $88 \%$ (AUC $=96 \%$ ), very similar to the RNN performance 254 (Accuracy $=86 \%, \mathrm{AUC}=95 \%$ ) and outperforming the $\mathrm{SNN}$ (Accuracy $=78 \%$, AUC=92\%). In comparison with 255 spontaneous EEG spectrograms, ERSPs provided better performance for all architectures. To assess the 256 performance of each individual channel, we also trained the DCNN with ERSP data from single channels and 
medRxiv preprint doi: https://doi.org/10.1101/19005611; this version posted September 11, 2019. The copyright holder for this preprint (which was not certified by peer review) is the author/funder, who has granted medRxiv a license to display the preprint in perpetuity.

All rights reserved. No reuse allowed without permission.

257 found that frontal (F3, Fz and F4) and parietal electrodes (P3, P4) provide the best performance compared to 258 frontopolar (Fp1, Fp2) electrodes (Figure 3c and 3d).

a) Neural Networks accuracy

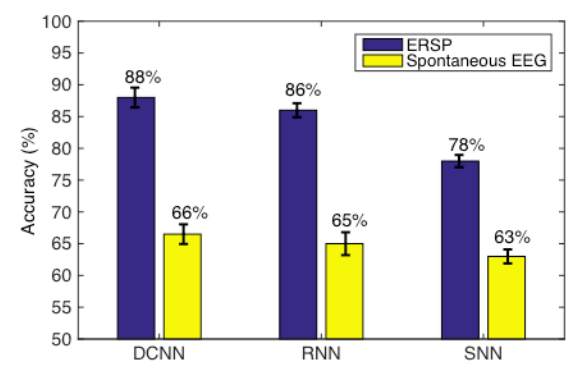

c) Electrode accuracy

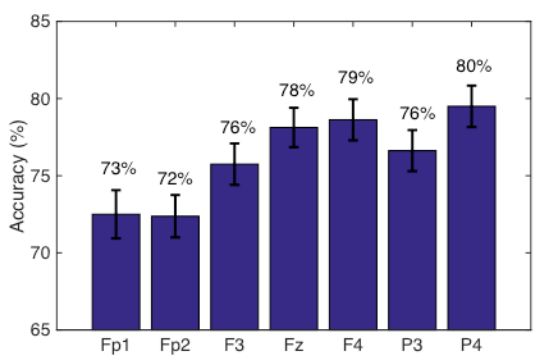

b) Neural Networks AUC

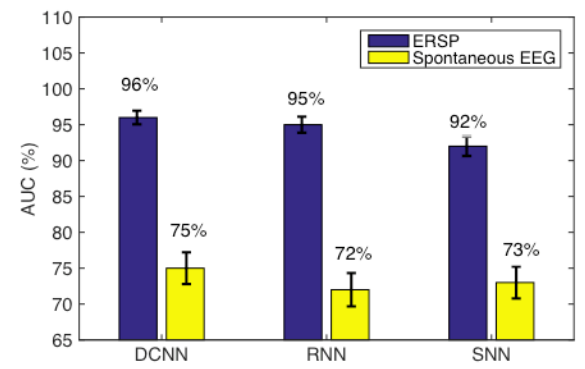

d) Electrode AUC

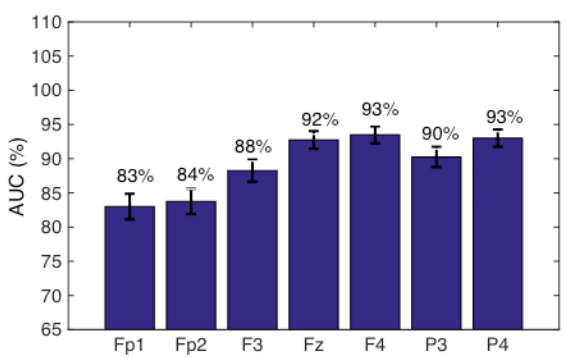

Figure 3. Performance assessment. Neural networks accuracy (a) and AUC (b) with ERSP and spontaneous EEG data.

Electrode accuracy (c) and AUC (d) in a DCNN trained with ERSP data from single channels. Error bars indicate mean square

263 error.

265 The mean DeepDream ERSP averaged over channels can be seen in Figure 4 (see Table S1 for individual

266 channels). The difference between groups reveals that the main feature that optimally excites the network nodes is

267 an increased power for the ADHD group in the delta-theta band (3-7 Hz) around $100 \mathrm{~ms}$ and a decreased power in

268 the alpha band $(7-12 \mathrm{~Hz})$ along the entire time course, with a residual decrease in theta and beta. Note that the

269 patterns shown in the DeepDream ERSP are very similar to the patterns of the ERSP computed from the real data

270 (Table S1), thus showing that the network is actually learning real neurophysiologically identifiable differences

271 between groups. 
a) $\mathrm{HC}$

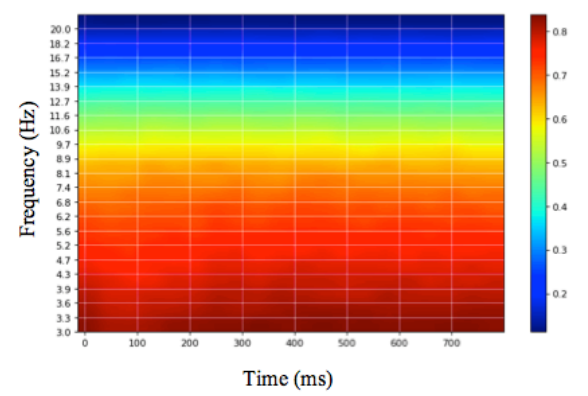

b) ADHD

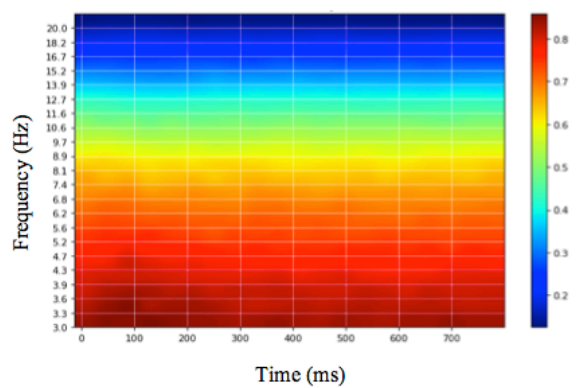

c) ADHD-HC

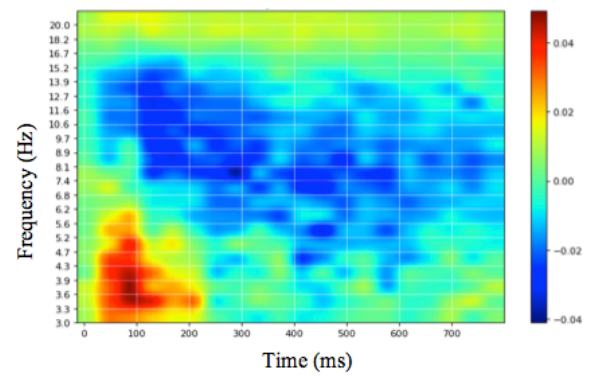

Figure 4. Mean DeepDream ERSP. Mean DeepDream ERSP averaged over channels generated after 400 experiments for healthy controls (a), ADHD patients (b) and their difference (c). Color bar units $=\mathrm{dB}$.

Behaviorally, the mean reaction time was significantly slower for $\mathrm{ADHD}$ compared to $\mathrm{HC}\left(\mathrm{RT}_{\mathrm{ADHD}}=368 \mathrm{~ms}\right.$,

$\left.277 \mathrm{RT}_{\mathrm{HC}}=321 \mathrm{~ms}, \beta=46 \mathrm{~ms}, \mathrm{CI}=[38,53] \mathrm{ms}, \mathrm{p}<0.001\right)$, which can be expected with this type of population with 278 attention deficits, but the mean percentage of responses for each group was not significantly different 279 (Accuracy $_{\mathrm{ADHD}}=62 \%$, Accuracy $\left._{\mathrm{HC}}=65 \%, \beta=0.12, \mathrm{CI}=[0.02,0.26], \mathrm{p}=0.10\right)$.

\section{$\underline{\text { Discussion }}$}

282 In this study we present a viable deep learning model for effective discrimination of patients with ADHD, 283 providing a new tool for the analysis of EEG dynamics in ADHD and supporting the potential of deep learning 284 strategies for biomarker development in neuropsychiatry. We deem this approach to be particularly interesting for 285 various reasons. First, it largely mitigates the need for EEG feature selection (spectral bands, time ranges, specific 286 ERP components and channels). Second, results with ERSPs represent an improvement over spontaneous EEG 287 spectrograms (e.g. subject Accuracy with DCNN was 88\% for ERSP vs. 66\% for spontaneous resting-state data).

288 Third, the performances of the proposed DCNN and RNN systems are very similar and they outperform the SNN 289 used for comparison. Finally, through the use of feature visualization, we identify neurophysiologically 290 interpretable features that can be extracted from the model, providing further validation and evidence that the 
291 network performance is not driven by noise or artifact signals in the data and providing a mechanistic model with

292 added value to understand pathophysiology.

294 The higher accuracy provided by DCNN and RNN compared to SNN proves that the complex deep approaches

295 with more layers and units provide better performance than more shallow networks. The similar performance of

296 DCNN compared to RNN shows, however, that the higher computational demands of RNN do not provide better

297 performance than the DCNN approaches, thus proving DCNN as a more efficient method than RNN.

299 The fact that ERSP data provide better performance than spontaneous EEG data with all architectures also shows

300 that event-related data from a highly yield task that elicits the primary executive functions impaired in ADHD is a

301 better predictor than spontaneous EEG data recorded while the participants are at resting state.

303 Finally, through the use of feature visualization we show that the main spectral features picked up by the DCNN

304 nodes are a decrease in alpha activity over the entire time course and an increased delta-theta activity around 100

$305 \mathrm{~ms}$ for ADHD patients compared to HC. There is evidence that an increased alpha activity (or alpha Event-Related

306 Synchronization, ERS) in conflict and inhibitory tasks is related with an improved inhibition of the prepotent

307 response, reflecting a top-down inhibitory control process ${ }^{41}$. Therefore, we interpret the decrease in alpha power in

308 ADHD as a deficit in cognitive control. On the other hand, the increased delta-theta activity is localized to $100 \mathrm{~ms}$

309 and is probably related to the increase in N100 amplitude in the time domain (Table S1). N100 is a visual sensory

310 evoked potential that is thought to index sensory analysis of simple stimulus features and whose amplitude is

311 influenced by selective attention ${ }^{42}$. The increased delta-theta power in that latency suggests that ADHD patients

312 manifest specific alterations in the process of early selection of visual task stimuli ${ }^{43}$. Given that there were no

313 significant differences in the percentage of correct responses between ADHD and HC, we interpret this as a

314 compensation strategy to offset inhibitory deficits by shifting more attention to the task ${ }^{44,45}$.

316 Note that the DeepDream spectrograms generated for Fp1 and Fp2 are substantially different and provide lower 317 performance that the other positions (F3, F4, Fz, P3, P4), which may be explained by the lower signal quality of 318 frontopolar positions due to blinks, muscle artefacts and sweat. The lower performance of F3 and P3 electrodes 319 compared to F4 and P4 may also be related to the lower power scale in their DeepDream spectrogram, respectively 
320 (Table S1). This may suggest the existence of inter-hemispheric differences in the features driving the 321 discrimination between ADHD and HC.

323 Similar studies have explored the application of deep learning to EEG signals. For example, DCNNs have been

324 used for epilepsy prediction and monitoring ${ }^{\star s}$, mental workload classification" and motor imagery classification"siss.

325 Deep neural networks have also shown convincing results in classifying psychiatric disorders such as dementia ${ }^{51}$

326 and $\mathrm{ADHD}^{52-57}$, mostly with MRI data. To our knowledge, this is the first study using a deep learning approach

327 with EEG event-related spectral data to discriminate adult ADHD patients from HC with no prior selection of

328 EEG features and the combination with feature visualization techniques to provide further mechanistic evidence of

329 the underlying pathophysiology driving the classification. This is particularly important, as it not only allows to

330 develop clinical tools but also to delineate pathological signatures and disease mechanisms.

332 One of the limitations of this study is the relatively small size of the dataset, with the consequent limitation on the 333 network due to susceptibility to overfitting. Although "early stopping" and regularization should mitigate this 334 issue, further improvements could be achieved with bigger datasets. Another limitation is the age difference 335 between the two groups. While the mean ages are well after the period of brain maturation when myelination and

336 ADHD symptoms are still changing, and well before a geriatric threshold when other type of biological changes 337 (including normal aging) may affect cognition, we addressed this possible confounder using Inverse Probability 338 Weighting. The age difference was an artifact caused by the fact that the two cohorts were recruited prospectively 339 for independent studies (though at the same time and with the same exact protocol and hardware) and then 340 analyzed together retrospectively to address the proposed questions, hence the lack of appropriately age-matched 341 controls. Future prospective validation studies should use larger cohorts and randomize age-matched controls.

343 It is also worth mentioning that, although the current work considerably eliminates the need for manual extraction 344 of features, it is still focused on classification during high yield incongruent trials of a specific task. While this 345 requires a priori knowledge constrains, if validated with higher definition EEG and bigger datasets, it may be a 346 helpful diagnostic and biomarker development strategy (i.e. choosing high yield events of a high yield task) with 347 practical future procedural advantages (i.e. it would be easy to implement it in clinical settings with currently 348 existing tools, such as tasks for neuropsychological assessments and standard EEG for electrophysiological 349 diagnosis). 
351 Our findings may also have several implications from the clinical perspective by bringing new information to

352 inform the clinician's decisions. Although the networks in this study have been trained with a small dataset of 40

353 subjects, if validated with bigger datasets this approach could be used to support the diagnosis of ADHD on a

354 single-patient basis. The fact that the current networks have been trained with low-resolution EEG datasets (7

355 channels) of short duration (3 minutes) would make it easy to implement them not only in an EEG clinical unit,

356 but possibly by an outpatient clinician, eliminating the need to get longer or higher quality data with sophisticated

357 and clinically unpractical EEG systems. However, even if these deep learning systems are properly validated in the

358 future, clinicians should view their output as statistical predictions, not as a ground truth, and they should judge

359 whether the prediction applies to that specific patient and decide if additional data or expertise is needed to inform

360 that decision.

362 Future work should include the exploration of this approach with larger datasets as well as a more systematic study

363 of network architecture and regularization schemes. This includes the use of deeper architectures, improved data

364 augmentation methods, alternative data segmentation and normalization schemes. With regards to data

365 preprocessing, we should consider improved spectral estimation using more advanced techniques such as state-

366 space estimation and multitapering ${ }^{58}$, and the use of cortical or scalp-mapped EEG data prior creation of 367 spectrograms.

369 Finally, we note that we make no attempt to fully-optimize our architecture in this study. In particular, no fine-

370 tuning of hyper-parameters has been carried out using a validation set approach, a task we reserve for future work

371 with larger datasets. Our aim was to validate the idea that deep learning approaches can provide value for the

372 analysis of time-frequency representations of EEG, and particularly ERSP data, for the effective discrimination of

373 ADHD.

\section{Data availability}

376 The datasets generated and analyzed during the current study are available from the senior author (JAC) on 377 reasonable request.

\section{Acknowledgements}

380 This research was partly supported by NIH grants (RO1 MH112737, R21 DA042271, R21 AG056958 and R21

381 MH113018) and the Louis V. Gerstner III Research Scholar Award to JAC. 


\section{Author contributions}

384 LD contributed with the processing of the data, the implementation of the deep learning systems, and the writing

385 of the article. GR contributed with the conception and design of the deep learning systems and the revision of the

386 manuscript. JC contributed with the conception and design of the study, the supervision of data acquisition and the

387 findings, and the critical revision of the manuscript. All authors gave the approval to the final version of the

388 manuscript to be published.

\section{Competing Interests}

391 LDV is an employee at Neuroelectrics and a PhD student in the Camprodon Lab. GR is a co-founder of

392 Neuroelectrics, a company that manufactures the EEG device used in this study. JAC is a member of the scientific

393 advisory board for Apex Neuroscience Inc.

\section{References}

1 Shamay-Tsoory, S. G. \& Aharon-Peretz, J. Dissociable prefrontal networks for cognitive and affective

4002 Biederman, J. et al. Functional impairments in adults with self-reports of diagnosed ADHD: A controlled 401 study of 1001 adults in the community. The Journal of clinical psychiatry 67, 524-540 (2006).

4023 Fayyad, J. et al. Cross-national prevalence and correlates of adult attention-deficit hyperactivity disorder.

403 The British journal of psychiatry : the journal of mental science 190, 402-409,

$404 \quad$ doi:10.1192/bjp.bp.106.034389 (2007).

4054 Kessler, R. C. et al. The prevalence and correlates of adult ADHD in the United States: results from the 406 National Comorbidity Survey Replication. The American journal of psychiatry 163, 716-723, 407 doi:10.1176/ajp.2006.163.4.716(2006).

4085 Polanczyk, G., de Lima, M. S., Horta, B. L., Biederman, J. \& Rohde, L. A. The worldwide prevalence of 409 ADHD: a systematic review and metaregression analysis. The American journal of psychiatry 164, 942410948 , doi:10.1176/ajp.2007.164.6.942(2007).

4116 Jasper, H. H., Solomon, P. \& Bradley, C. Electroencephalographic analyses of behavior problem 412 children. American Journal of Psychiatry 95, 641-658, doi:10.1176/ajp.95.3.641 (1938). 
4137 Lenartowicz, A. \& Loo, S. K. Use of EEG to Diagnose ADHD. Current psychiatry reports 16, 498, 414 doi:10.1007/s11920-014-0498-0 (2014).

4158 Fabiani, M., Gratton, G. \& Federmeier, K. D. in Handbook of psychophysiology, 3rd ed. 85-119 416 (Cambridge University Press, 2007).

4179 Sanei, S. \& Chambers, J. A. EEG Signal Processing. (John Wiley \& Sons Ltd, 2013).

$418 \quad 10 \quad$ Woodman, G. F. A brief introduction to the use of event-related potentials in studies of perception and 419 attention. Attention, perception \& psychophysics 72, 2031-2046, doi:10.3758/app.72.8.2031 (2010).

42011 Pokryszko-Dragan, A. et al. Event-related potentials and cognitive performance in multiple sclerosis 421 patients with fatigue. Neurological sciences : official journal of the Italian Neurological Society and of 422 the Italian Society of Clinical Neurophysiology 37, 1545-1556, doi:10.1007/s10072-016-2622-x (2016).

42312 Paulraj, M. P., Subramaniam, K., Yaccob, S. B., Adom, A. H. \& Hema, C. R. Auditory evoked potential 424 response and hearing loss: a review. The open biomedical engineering journal 9, 17-24,

425 doi:10.2174/1874120701509010017 (2015).

42613 Durstewitz, D., Koppe, G. \& Meyer-Lindenberg, A. Deep neural networks in psychiatry. Molecular 427 Psychiatry, doi:10.1038/s41380-019-0365-9 (2019).

42814 Kroupi, E. et al. in HBP Student Conference - Transdisciplinary Research Linking Neuroscience, Brain $429 \quad$ Medicine and Computer Science (Viena, Austria, 2017).

43015 Ruffini, G., Ibañez, D., Castellano, M., Dunne, S. \& Soria-Frisch, A. EEG-driven RNN Classification for 431 Prognosis of Neurodegeneration in At-Risk Patients. Artificial Neural Networks and Machine Learning432 ICANN 2016, 306-313 (2016).

43316 Goodfellow, I., Bengio, Y. \& Courville, A. Deep Learning. (MIT Press, 2016).

43417 Mueller, A., Candrian, G., Kropotov, J. D., Ponomarev, V. A. \& Baschera, G. M. Classification of 435 ADHD patients on the basis of independent ERP components using a machine learning system. Nonlinear 436 Biomedical Physics 4, S1, doi:10.1186/1753-4631-4-s1-s1 (2010).

43718 Tenev, A. et al. Machine learning approach for classification of ADHD adults. International journal of 438 psychophysiology : official journal of the International Organization of Psychophysiology 93, 162-166, 439 doi:10.1016/j.ijpsycho.2013.01.008 (2014).

44019 Jahanshahloo, H. R., Shamsi, M., Ghasemi, E. \& Kouhi, A. Automated and ERP-Based Diagnosis of 441 Attention-Deficit Hyperactivity Disorder in Children. Journal of Medical Signals and Sensors 7, 26-32 $442 \quad$ (2017). 
44320 Nazhvani, A. D., Boostani, R., Afrasiabi, S. \& Sadatnezhad, K. Classification of ADHD and BMD

444 patients using visual evoked potential. Clinical neurology and neurosurgery 115, 2329-2335,

445 doi:10.1016/j.clineuro.2013.08.009 (2013).

$44621 \quad$ Sadatnezhad, K., Boostani, R. \& Ghanizadeh, A. Classification of BMD and ADHD patients using their

447 EEG signals. Expert Systems with Applications 38, 1956-1963,

448 doi:https://doi.org/10.1016/j.eswa.2010.07.128 (2011).

44922 Ahmadlou, M. \& Adeli, H. Wavelet-synchronization methodology: a new approach for EEG-based 450 diagnosis of ADHD. Clinical EEG and neuroscience 41, 1-10, doi:10.1177/155005941004100103

$451 \quad$ (2010).

$452 \quad 23$ Abibullaev, B. \& An, J. Decision support algorithm for diagnosis of ADHD using

453 electroencephalograms. Journal of medical systems 36, 2675-2688, doi:10.1007/s10916-011-9742-x

$454 \quad$ (2012).

45524 Ruffini, G. et al. Algorithmic complexity of EEG for prognosis of neurodegeneration in idiopathic rapid 456 eye movement behavior disorder (RBD). bioRxiv (2018).

45725 Oord, A. v. d., Dieleman, S. \& Schrauwen, B. in Proceedings of the 26th International Conference on 458 Neural Information Processing Systems - Volume 2 2643-2651 (Curran Associates Inc., Lake Tahoe, $459 \quad$ Nevada, 2013).

46026 Tsinalis, O., M. Matthews, P., Guo, Y. \& Zafeiriou, S. Automatic Sleep Stage Scoring with Single$461 \quad$ Channel EEG Using Convolutional Neural Networks. (2016).

46227 Vilamala, A., Madsen, K. H. \& Hansen, L. K. Deep Convolutional Neural Networks for Interpretable 463 Analysis of EEG Sleep Stage Scoring. 2017 International workshop on Machine Learning for signal $464 \quad$ processing (2017).

46528 Ruffini, G. et al. Deep Learning With EEG Spectrograms in Rapid Eye Movement Behavior Disorder. $466 \quad$ Frontiers in neurology 10, 806 (2019).

46729 Dauwels, J., Vialatte, F., Musha, T. \& Cichocki, A. A comparative study of synchrony measures for the 468 early diagnosis of Alzheimer's disease based on EEG. NeuroImage 49, 668-693, 469 doi:https://doi.org/10.1016/j.neuroimage.2009.06.056 (2010).

$47030 \quad$ Alexander, M., Christopher, O. \& Mike, T. Inceptionism: Going Deeper into Neural Networks. Google $471 \quad$ Research Blog (2015). 
$47231 \quad$ American-Psychiatric-Association. The Diagnostic and Statistical Manual of Mental Disorders. 5th

473 Edition edn, (American Psychiatric Publishing, 2013).

$47432 \quad$ Kessler, R. C. et al. The World Health Organization Adult ADHD Self-Report Scale (ASRS): a short

475 screening scale for use in the general population. Psychol Med 35, 245-256 (2005).

47633 Kopp, B., Rist, F. \& Mattler, U. N200 in the Flanker task as a neurobehavioral tool for investigating

477 executive control. Psychophysiology 33, 282-294 (1996).

47834 Abadi, M. et al. in Proceedings of the 12th USENIX conference on Operating Systems Design and

479 Implementation 265-283 (USENIX Association, Savannah, GA, USA, 2016).

48035 Srivastava, N., Hinton, G., Krizhevsky, A., Sutskever, I. \& Salakhutdinov, R. Dropout: A Simple Way to

481 Prevent Neural Networks from Overfitting. Journal of Machine Learning Research 15, 1929-1958

$482 \quad$ (2014).

$483 \quad 36 \quad$ Prechelt, L. in Neural Networks: Tricks of the Trade (eds Genevieve B. Orr \& Klaus-Robert Müller) 484 55-69 (Springer Berlin Heidelberg, 1998).

48537 Hochreiter, S. \& Schmidhuber, J. Long Short-Term Memory. Neural Computation 9, 1735-1780, 486 doi:10.1162/neco.1997.9.8.1735 (1997).

48738 Antti, A., Tapio, P., Willem, W., Bernard De, B. \& Tapio, S. 3-13 (PMLR, 2009).

48839 Linn, K. A., Gaonkar, B., Doshi, J., Davatzikos, C. \& Shinohara, R. T. Addressing Confounding in 489 Predictive Models with an Application to Neuroimaging. The international journal of biostatistics 12, 31$490 \quad 44$, doi:10.1515/ijb-2015-0030 (2016).

$49140 \quad$ Austin, P. C. An Introduction to Propensity Score Methods for Reducing the Effects of Confounding in 492 Observational Studies. Multivariate behavioral research 46, 399-424, 493 doi:10.1080/00273171.2011.568786 (2011).

$494 \quad 41 \quad$ Klimesch, W., Sauseng, P. \& Hanslmayr, S. EEG alpha oscillations: The inhibition-timing hypothesis. 495 Brain Research Reviews 53, 63-88, doi:https://doi.org/10.1016/j.brainresrev.2006.06.003 496 (2007).

$49742 \quad$ Rugg, M. D., Milner, A. D., Lines, C. R. \& Phalp, R. Modulation of visual event-related potentials by 498 spatial and non-spatial visual selective attention. Neuropsychologia 25, 85-96 (1987).

49943 Yordanova, J., Heinrich, H., Kolev, V. \& Rothenberger, A. Increased event-related theta activity as a 500 psychophysiological marker of comorbidity in children with tics and attention-deficit/hyperactivity 
501

502

50344

504

505

506

$507 \quad 45$

508

$509 \quad 46$

510

$511 \quad 47$

512

51348

514

$515 \quad 49$

516

$517 \quad 50$

518

$519 \quad 51$

520

521

$522 \quad 52$

523

524

525

$526 \quad 53$

527

$528 \quad 54$

529

$530 \quad 55$

disorders. NeuroImage 32, 940-955,

doi:https://doi.org/10.1016/j.neuroimage.2006.03.056 (2006).

Broyd, S. J. et al. The effect of methylphenidate on response inhibition and the event-related potential of children with attention deficit/hyperactivity disorder. International journal of psychophysiology : official journal of the International Organization of Psychophysiology 58, 47-58, doi:10.1016/j.ijpsycho.2005.03.008 (2005).

Prox, V., Dietrich, D. E., Zhang, Y., Emrich, H. M. \& Ohlmeier, M. D. Attentional processing in adults with ADHD as reflected by event-related potentials. Neuroscience Letters 419, 236-241 (2007).

\section{Liang, J., Lu, R., Zhang, C. \& Wang, F. in IEEE International Conference on Healthcare Informatics} (ICHI) 184-191 (2016).

Ma, T. et al. The extraction of motion-onset VEP BCI features based on deep learning and compressed sensing. J Neurosci Methods 275, 80-92, doi:10.1016/j.jneumeth.2016.11.002 (2017).

Bashivan, P., Rish, I., Yeasin, M. \& Codella, N. Learning Representations from EEG with Deep Recurrent-Convolutional Neural Networks. (2015).

Tabar, Y. R. \& Halici, U. A novel deep learning approach for classification of EEG motor imagery signals. J Neural Eng 14, 016003, doi:10.1088/1741-2560/14/1/016003 (2017).

An, X., Kuang, D., Guo, X., Zhao, Y. \& He, L. in Intelligent Computing in Bioinformatics. (eds DeShuang Huang, Kyungsook Han, \& Michael Gromiha) 203-210 (Springer International Publishing).

Vieira, S., Pinaya, W. H. \& Mechelli, A. Using deep learning to investigate the neuroimaging correlates of psychiatric and neurological disorders: Methods and applications. Neurosci Biobehav Rev 74, 58-75, doi:10.1016/j.neubiorev.2017.01.002 (2017).

52 Deshpande, G., Wang, P., Rangaprakash, D. \& Wilamowski, B. Fully Connected Cascade Artificial Neural Network Architecture for Attention Deficit Hyperactivity Disorder Classification From Functional Magnetic Resonance Imaging Data. IEEE transactions on cybernetics 45, 2668-2679, doi:10.1109/tcyb.2014.2379621 (2015).

53 Han, X., Zhong, Y., He, L., Yu, P. S. \& Zhang, L. in Brain Informatics and Health. (eds Yike Guo et al.) 156-166 (Springer International Publishing).

54 Hao, A. J., He, B. L. \& Yin, C. H. in 2015 IET International Conference on Biomedical Image and Signal Processing (ICBISP 2015). 1-6.

55 Kuang, D. \& He, L. in 2014 International Conference on Cloud Computing and Big Data. 27-32. 
medRxiv preprint doi: https://doi.org/10.1101/19005611; this version posted September 11, 2019. The copyright holder for this preprint (which was not certified by peer review) is the author/funder, who has granted medRxiv a license to display the preprint in perpetuity.

All rights reserved. No reuse allowed without permission.

$53156 \quad$ Kuang, D., Guo, X., An, X., Zhao, Y. \& He, L. in Intelligent Computing in Bioinformatics. (eds De-

532 Shuang Huang, Kyungsook Han, \& Michael Gromiha) 225-232 (Springer International Publishing).

$53357 \quad$ Zou, L., Zheng, J., Miao, C., Mckeown, M. J. \& Wang, Z. J. 3D CNN Based Automatic Diagnosis of

534 Attention Deficit Hyperactivity Disorder Using Functional and Structural MRI. IEEE Access 5, 23626-

535 23636, doi:10.1109/ACCESS.2017.2762703 (2017).

$53658 \quad$ Kim, S.-E., Behr, M. K., Ba, D. \& Brown, E. N. State-space multitaper time-frequency analysis.

$537 \quad$ Proceedings of the National Academy of Sciences 115, E5, doi:10.1073/pnas.1702877115 (2018).

538 